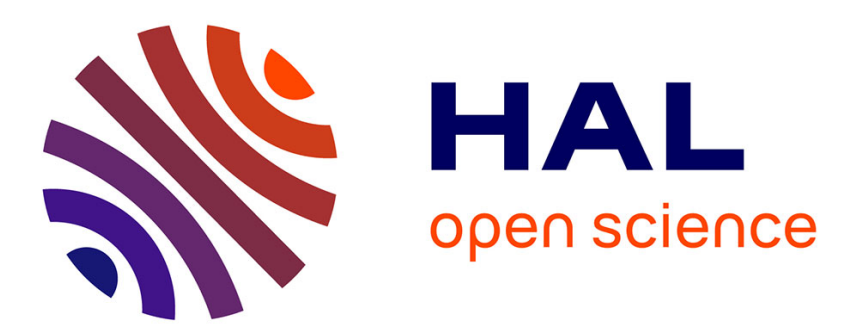

\title{
Drag measurements in laterally confined 2D canopies: reconfiguration and sheltering effect
}

Sylvie Barsu, Delphine Doppler, J. John Soundar Jerome, Nicolas Riviere, Michel Lance

\section{- To cite this version:}

Sylvie Barsu, Delphine Doppler, J. John Soundar Jerome, Nicolas Riviere, Michel Lance. Drag measurements in laterally confined 2D canopies: reconfiguration and sheltering effect. Physics of Fluids, 2016, 28 (10), pp.107101. 10.1063/1.4962309 . hal-01743945v2

\section{HAL Id: hal-01743945 \\ https://hal.science/hal-01743945v2}

Submitted on 9 Apr 2018

HAL is a multi-disciplinary open access archive for the deposit and dissemination of scientific research documents, whether they are published or not. The documents may come from teaching and research institutions in France or abroad, or from public or private research centers.
L'archive ouverte pluridisciplinaire HAL, est destinée au dépôt et à la diffusion de documents scientifiques de niveau recherche, publiés ou non, émanant des établissements d'enseignement et de recherche français ou étrangers, des laboratoires publics ou privés. 


\title{
Drag measurements in laterally confined 2D canopies: Reconfiguration and sheltering effect
}

\author{
Sylvie Barsu, Delphine Doppler, ${ }^{\text {a) }}$ J. John Soundar Jerome, Nicolas Rivière, \\ and Michel Lance \\ Univ Lyon, Université Claude Bernard Lyon 1, INSA de Lyon, École Centrale \\ de Lyon, CNRS, LMFA UMR 5509, 41 Boulevard Latarjet, F-69622 Villeurbanne Cedex, \\ Lyon, France
}

(Received 2 May 2016; accepted 23 August 2016; published online 4 October 2016)

\begin{abstract}
Plants in aquatic canopies deform when subjected to a water flow and so, unlike a rigid bluff body, the resulting drag force $F_{D}$ grows sub-quadratically with the flow velocity $\bar{U}$. In this article, the effect of density on the canopy reconfiguration and the corresponding drag reduction is experimentally investigated for simple 2D synthetic canopies in an inclinable, narrow water channel. The drag acting on the canopy, and also on individual sheets, is systematically measured via two independent techniques. Simultaneous drag and reconfiguration measurements demonstrate that data for different Reynolds numbers (400-2200), irrespective of sheet width $(w)$ and canopy spacing $(\ell)$, collapse on a unique curve given by a bending beam model which relates the reconfiguration number and a properly rescaled Cauchy number. Strikingly, the measured Vogel exponent $\mathcal{V}$ and hence the drag reduction via reconfiguration is found to be independent of the spacing between sheets and the lateral confinement; only the drag coefficient decreases linearly with the sheet spacing since a strong sheltering effect exists as long as the spacing is smaller than a critical value depending on the sheet width. Published by AIP Publishing. [http://dx.doi.org/10.1063/1.4962309]
\end{abstract}

\section{INTRODUCTION}

Vegetation in rivers is often considered as a source of water resistance which slows down the water conveyance. ${ }^{1}$ It is also one of the main components for river equilibrium, insofar as it prevents body erosion by providing bed stabilization, it plays a vital role during floods. It is crucial for sediment transport, water quality, and also shelter to provide the necessary habitat for the biodiversity of aquatic species. ${ }^{2}$ It is then useful to understand the mechanical behaviour of aquatic canopies resulting from the interaction between vegetation and a water flow. From land-use planning to river management, ${ }^{3}$ such a knowledge would also shed light upon plant biomechanics ${ }^{4,5}$ and improve bio-inspired engineering. ${ }^{6,7}$

Traditionally, studies on aquatic vegetation explored its influence on flow properties, like velocity distribution, wake dynamics, turbulence, ${ }^{8-10}$ water conveyance, and sediment transport ${ }^{11-13}$ by considering it simply as a rigid or flexible roughness element. So far, such works describe qualitatively and quantitatively the role of real plants ${ }^{1,14}$ and rigid ${ }^{15}$ or flexible ${ }^{16-18}$ artificial canopies as an active element on flow friction losses. Recent investigations also explore various mechanically activated phenomena in plants or plant canopies that arise from interaction between the fluid flow (air or water) and vegetation. ${ }^{19}$ For example, flow-induced vibration and the origin of coherent structures on crop canopies, ${ }^{20-23}$ dissipation of wave energy, ${ }^{24,25}$ population growth and ecological consequences, ${ }^{26}$ flow-triggered pruning, ${ }^{27}$ and seed-dispersal ${ }^{28,29}$ were studied.

For many such investigations, one of the key ingredients in the analysis is the drag force experienced by a canopy, a plant, and its parts. The profile drag exerted by a flow on a bluff body

\footnotetext{
a)Electronic mail: delphine.doppler@univ-lyon1.fr
} 
(rigid or flexible) is proportional to the frontal area $A_{\mathrm{s}}$ exposed to the flow and to the square of the flow velocity $\bar{U}$ such that the profile drag coefficient

$$
C_{D}=\frac{F_{D}}{\frac{1}{2} \rho U^{2} A_{s}},
$$

where $A_{s}$ is independent of the flow velocity for rigid objects. For flexible objects, however, the frontal area $A_{\mathrm{s}}$ can reduce drastically due to bending and/or twisting. In this context, Vogel ${ }^{30-32}$ studied the deformation of tree leaves subjected to air flow and elucidated the resulting drag reduction mechanism. In fact, the shape of a tree leaf (or any flexible object) is the result of an equilibrium between drag $F_{\mathrm{D}}$ and elastic restoring forces $F_{\mathrm{E}}$. Thereby, the reconfiguration of a flexible body (and hence, $A_{S}$ ) is, in general, a function of the non-dimensional Cauchy number $\left(C_{\mathrm{Y}}=F_{\mathrm{D}} / F_{\mathrm{E}}\right)$ and the boundary conditions. ${ }^{33}$ Numerous experimental and numerical investigations then considered such reconfiguration effects in various systems. The effect of wind velocity on drag was measured on real trees and leaves. ${ }^{31,34-37}$ Analogous artificial systems were studied, including numerical models which reproduce experimental measurements on a fibre subjected to a soap film, ${ }^{38,39}$ a flexible plate in air $^{40}$ or water ${ }^{41,42}$ flow, and artificial leaves. ${ }^{43,44}$ Experimental data are observed to collapse on a single reconfiguration curve so that $A_{s} \propto C_{\mathrm{Y}}^{V / 2}$, where $\mathcal{V}$ is the so called Vogel exponent. ${ }^{31}$ Therefore, for given physical properties of the fluid and the flexible body, the frontal area $A_{s}$ is only a function of the flow velocity $\bar{U}$ and so the drag reduction can be understood by introducing the Vogel exponent $\mathcal{V}$. The drag force is then

$$
F_{D} \propto \frac{1}{2} \rho U^{2+v} A,
$$

where $\mathrm{A}$ is the undeformed frontal area so that $\mathcal{V}=0$ for a rigid body and, in general, $\mathcal{V}<0$, for a flexible body. $4,38,42,43,45$

However, in rivers, canopies are sets of plants close to each other, giving rise to a strong screening effect, so that plants in canopies do not behave like single plants ${ }^{46,47}$ There is also an increasing interest in theoretical models for drag force acting on poro-elastic systems. ${ }^{19,21,48-51}$ Few studies make explicit the influence of canopy density on drag. Through theoretical modelling, Gosselin and de Langre ${ }^{52}$ investigated the effect of surface density on the Vogel exponent of a synthetic poro-elastic system. They considered a ball covered-up by filaments analogous to coniferous trees to understand its reconfiguration due to a fluid flow through a porous medium. Depending on the number of filaments per unit length, the Vogel exponent is shown to vary between $-2 / 3$ and -1 . In Thom ${ }^{46}$ the effective drag force is measured on an artificial canopy in a wind channel and it is found to be much smaller than the expected value obtained by adding individual drag forces on each roughness element taken separately. This sheltering effect is also observed in other configurations: flexible stalks ${ }^{53}$ or rigid cylinders and trees ${ }^{54}$ in air. In water, the trend is not so obvious (see Peterson et al. ${ }^{55}$ ). Nepf ${ }^{15}$ and Poggi et al. ${ }^{56}$ show opposite trends for the drag coefficients evolution with density. Recent numerical work by Leclercq and de Langre ${ }^{57}$ shows that the Vogel exponent $\mathcal{V}$ can depend on the incident velocity profile. In a dense canopy, it is expected that the velocity field around the sheets depends on the canopy spacing. Therefore it is not straightforward how plant density and confinement would affect deformation of individual plants in a canopy. In addition, to the authors' knowledge, there exists no simultaneous experimental measurements of drag and reconfiguration in submerged canopies as a function of different canopy densities.

In this context, it is the objective of this article to investigate the drag force acting on an aquatic canopy with respect to flow velocity, canopy densities, and lateral confinement. In order to establish the relationship between the global drag force acting on the canopy and the resulting canopy reconfiguration and then to elucidate the role of canopy density, it is important to measure the drag force and the sheet deflection independently for various sheet densities and confinements. The focus is put on a simple quasi-2D porous media made up of an array of flexible flat plates aligned in the direction of the flow and confined laterally by channel walls. Independent techniques to measure the sheet deflection and the sheet drag force are first developed. Drag reduction via reconfiguration and sheltering effects is then explored experimentally. 


\section{SIMULTANEOUS RECONFIGURATION AND DRAG MEASUREMENTS}

\section{A. Experimental setup}

The experimental setup consists of a narrow open channel (length $L=2 \mathrm{~m}$ and width $b=$ $40 \mathrm{~mm}$ ). Its slope $\beta$ is adjustable from horizontal $\left(0^{\circ}\right)$ to $2.7^{\circ}$ with a precision of about $0.1 \mathrm{~mm} / \mathrm{m}$. The flow rate $Q$ at the channel entrance can be controlled using a variable area flow meter $\left(200-14001 \mathrm{~h}^{-1}\right)$. Thus, by measuring the mean water height $h_{\mathrm{w}}$ with a stream gauge, the mean flow velocity $\bar{U}=Q / b h_{\mathrm{w}}$ is known. The canopy consists of thin PVC transparent strips (thickness $e=0.1 \mathrm{~mm}$, height $h=60 \mathrm{~mm}$, width $w=10,15$, or $20 \mathrm{~mm}$ ) embedded on LEGO $囚$ bricks so that the spacing between each sheet, $\ell$, can systematically be varied (about 10 different spacings were tested for each sheet width, from $\ell=8$ to $160 \mathrm{~mm}$ ). This canopy of PVC sheets is fixed to the bottom of the hydraulic channel as illustrated in the schematic (Fig. 1). The canopy occupies the entire channel length. The mechanical properties of each PVC sheet, namely, the physical dimensions, density $\left(\rho=1.41 \times 10^{3} \mathrm{~kg} \mathrm{~m}^{-3}\right)$, and elasticity modulus $(E=3.78 \mathrm{GPa})$ are measured before hand using a digital calliper, analytical scales, and the tensile tests on dynamic mechanical analysis machine Q800 from TA instruments, respectively.

The aim of the setup is to simultaneously measure drag force and the sheet deflection for various canopy densities as a function of the water velocity. The former is accomplished by two independent techniques: (1) via the pressure drop across the channel and (2) via direct observation of individual sheet reconfiguration.

\section{B. Sheet drag via deflection measurement}

At any chosen flow rate, the water height can be maintained a constant at the canopy top level by carefully adjusting the outlet valve and channel inclination $\beta$. Once a steady flow is established in the channel, all sheets are observed to bend in the same manner and they are almost motionless. Only very small-amplitude oscillations at natural frequency sometimes occur when the setup is exposed to vibrations mainly due to the pump irregularities. For each sheet, the restoring bending force should then be balanced by the normal drag force on the sheet, ${ }^{40,58}$

$$
-E I \frac{\partial^{3} \varphi}{\partial s^{3}}=f_{N}(s),
$$

where $f_{N}(s)$ is sheet drag force per unit height of the sheet, $s$ is the coordinate along the beam, $\varphi(s)$ the local beam deflection (see left inset Fig. 1), $e$ is the sheet thickness $(0.1 \mathrm{~mm})$, and $I=w e^{3} / 12$ is

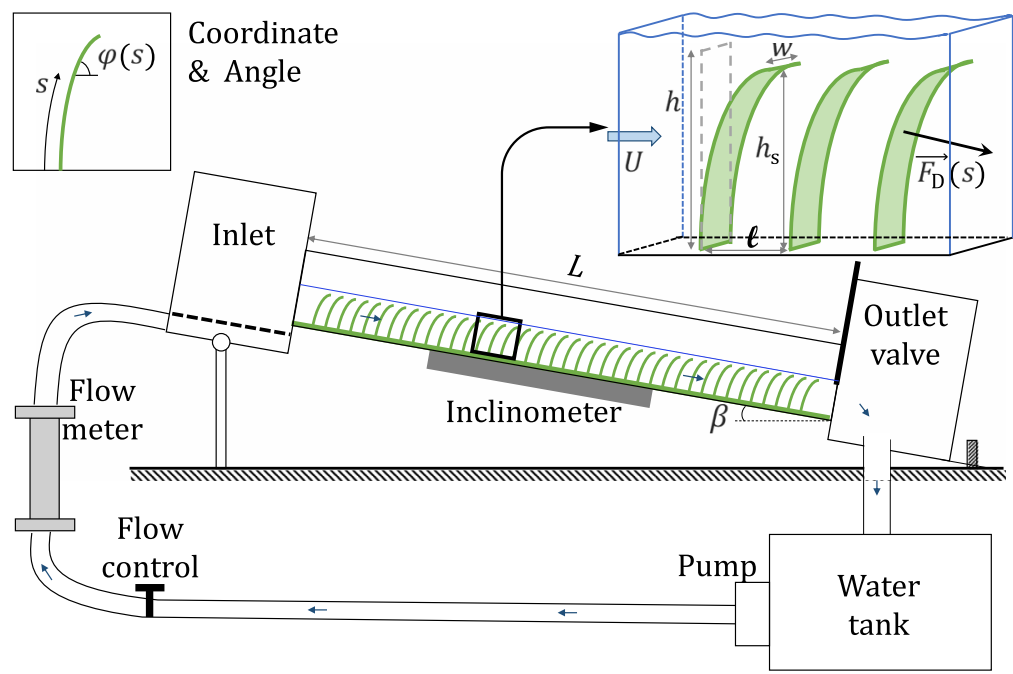

FIG. 1. The experimental setup. The open channel has an adjustable water flow rate $Q$ and inclination angle $\beta$. An array of PVC sheets representing an aquatic canopy is fixed all along the channel. The outlet valve is set to adjust the water height $h_{\mathrm{w}}$. 


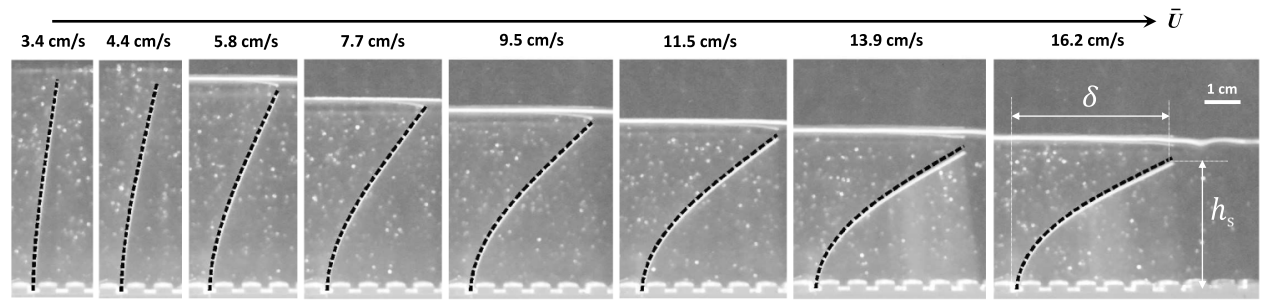

FIG. 2. Comparison between individual sheet deflection (thick white line) and non-linear bending beam model (dashed black line) for different mean flow velocities $\bar{U}$. The Cauchy number $C_{\mathrm{Y}}$ (Eq. (4)) is adjusted until a close fit is obtained. Thus, an equivalent drag coefficient is calculated: the local drag coefficient $C_{\mathrm{D}}^{l}$.

the sheet quadratic moment. The boundary conditions are applied at the extremities, embedded for $s=0$ and free for $s=h$, respectively, $\varphi(0)=\pi / 2$ and $\partial_{s} \varphi(h)=\partial_{s}^{2} \varphi(h)=0$. If $\bar{U} \sin \varphi(s)$ is the local incident flow speed on the sheet and $\tilde{s}=s / h$ is the non-dimensional coordinate along the beam, Eq. (3) becomes

$$
\frac{\partial^{3} \varphi}{\partial \tilde{s}^{3}}=-C_{\mathrm{Y}}^{l} \sin ^{2} \varphi(\tilde{s})
$$

where $C_{\mathrm{Y}}^{l}=C_{\mathrm{D}}^{l} \rho w h^{3} \bar{U}^{2} / 2 E I$ is the Cauchy number and the local drag coefficient $C_{\mathrm{D}}^{l}$ is defined, based on the local sheet drag $F_{D}^{l}$, as $C_{D}^{l}=F_{D}^{l} / \frac{1}{2} \rho \bar{U}^{2} h_{s} w$.

Using a planar LASER sheet, we can visualize the deflection of individual PVC sheets in the canopy (see thick white lines in Fig. 2). Thus, the sheet deflection $\delta$ and height $h_{\mathrm{s}}$ are directly measured with an accuracy of $1 \mathrm{~mm}$. Using $\delta$ and $h_{\mathrm{s}}$ in the solution of the bending beam model, an equivalent Cauchy number $C_{\mathrm{Y}}^{l}$ can be computed, so that the computed sheet profile (dashed black lines) matches with the observed sheet reconfiguration as shown in Fig. 2. Knowing the sheet physical properties and the mean flow velocity, the local sheet drag force $F_{\mathrm{D}}^{l}$ is then determined.

Figures 3(a) and 3(b) show typical variations of the local sheet drag force $F_{\mathrm{D}}^{l}$ with respect to flow velocity $\bar{U}$ when the sheet width $w=10 \mathrm{~mm}$ and $20 \mathrm{~mm}$, respectively. Each data point is an average drag force over 9-12 sheets in the canopy (depending on the run considered). Different symbols represent various measurements for a wide range of sheet spacings $\ell$. For any given canopy density, $F_{\mathrm{D}}^{l}$ is observed to increase, in general, monotonically with water velocity $\bar{U}$. At a fixed velocity $\bar{U}$, the drag force is observed to be smaller for a denser canopy. In fact, Fig. 3(b) shows that $F_{\mathrm{D}}^{l}$ can be stronger by an order of magnitude if the distance between sheets $\ell$ is much larger than the sheet width $w$. When the sheet spacing is sufficiently large, i.e., $\ell \geqslant 32 \mathrm{~mm}$ and $\ell \geqslant 56 \mathrm{~mm}$ for sheet widths $w=10 \mathrm{~mm}$ and $w=20 \mathrm{~mm}$, respectively, the measured drag force data collapse onto a leading curve given by $F_{\mathrm{D}}^{l} \propto \bar{U}^{2}$. On the other hand, if the canopy is dense, the drag force data do not collapse and in particular, they show a sub-quadratic variation, as inferred by Vogel for isolated elements. ${ }^{30-32}$ This can be verified in Figure 4(a) where the same data as Figure 3(b) are shown in a $\log -\log$ plot. Two regimes appear, namely, "rigid" (small $\bar{U}, F_{\mathrm{D}}^{l} \propto \bar{U}^{2}$, continuous line) and "elastic" (large $\bar{U}, F_{\mathrm{D}}^{l} \propto \bar{U}^{2+\mathcal{V}}$, dashed line).

\section{Sheet drag via an inclinometer}

Once a steady flow is established in the channel, the drop in pressure across the channel must be entirely compensated by wall friction losses and the global drag force experienced by the canopy. Moreover, the contribution of the channel skin friction is small compared to the net profile drag for the Reynolds numbers studied here (see, for example, Temple et al. ${ }^{59}$ or Nepf and Vivoni ${ }^{47}$ ). The classical Darcy-Weisbach formula used to estimate the pressure drop due to skin friction gives a maximal slope about $1 \mathrm{~mm} / \mathrm{m}$, which is of the order of the inclinometer precision. Therefore, the gravity force should balance the head loss due to the presence of a canopy. Thereby, a global measure of the canopy drag can be obtained as done by Wu et al. ${ }^{16}$ If $\beta$ is the channel inclination angle and $A_{\mathrm{c}}=b h_{\mathrm{w}}$ is the channel cross-sectional area, the pressure drop should be $\rho g L \sin \beta A_{\mathrm{c}}$. 
(a) Sheet width $w=10 \mathrm{~mm}$, confinement $w / b=25 \%$
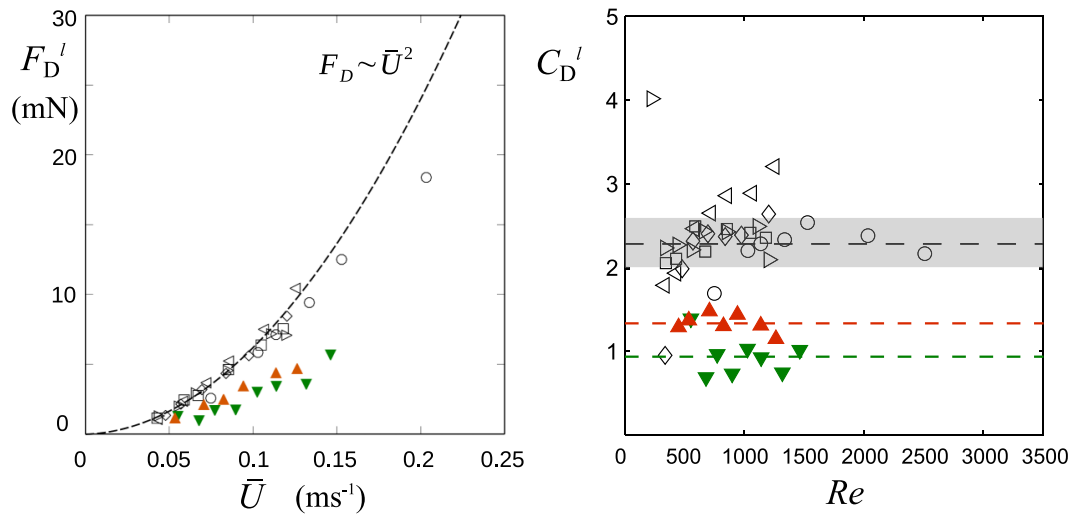

Sheet spacing
\begin{tabular}{cc}
$l$ & $/ w$ \\
\hline$\nabla$ & 0.8 \\
$\Delta$ & 2.4 \\
$\circ$ & 3.2 \\
$\square$ & 4.8 \\
$\triangleright$ & 7.2 \\
$\diamond$ & 9.6 \\
$\triangleleft$ & 14.4 \\
\hline
\end{tabular}

(b) Sheet width $w=20 \mathrm{~mm}$, confinement $w / b=50 \%$
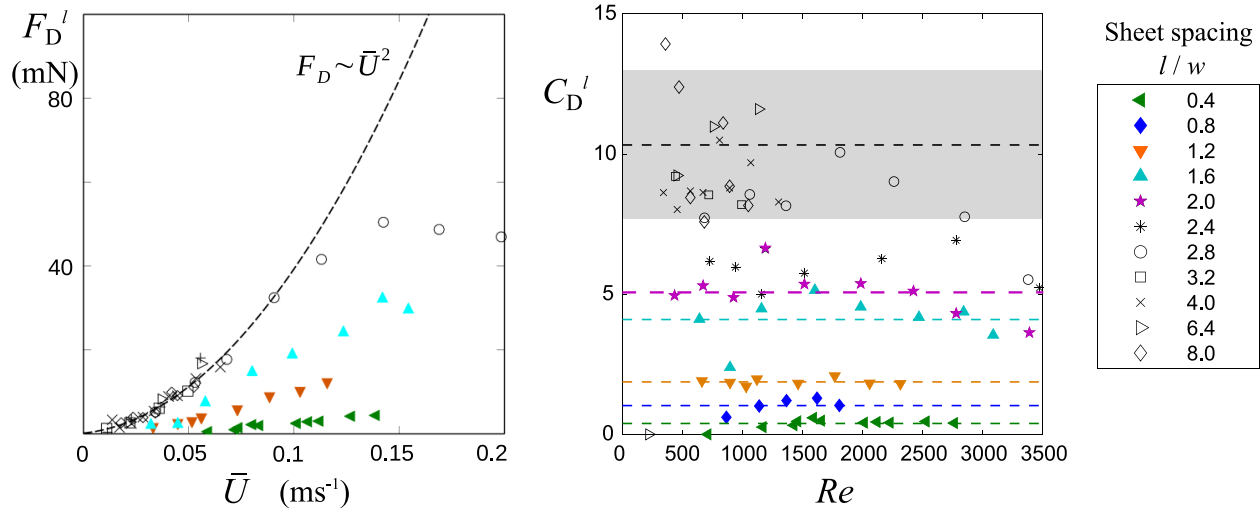

FIG. 3. On the left, the sheet drag force $F_{\mathrm{D}}^{l}$ (in $\mathrm{mN}$ ) is plotted as a function of the water velocity $\bar{U}\left(\mathrm{~ms}^{-1}\right)$ for various canopy densities. The sheet drag is deduced from the force required to produce the measured sheet deflection. The dashed line where all data above a critical sheet spacing collapse is a quadratic fit $\left(F_{\mathrm{D}}^{l} \propto \bar{U}^{2}\right)$ to the data listed in the inset. On the right, a non-dimensional plot of the same data. The mean values are computed for the different series (dashed lines) and for plateau value. The gray area represents the standard deviation for this plateau value.

(a) Power laws for the local drag force

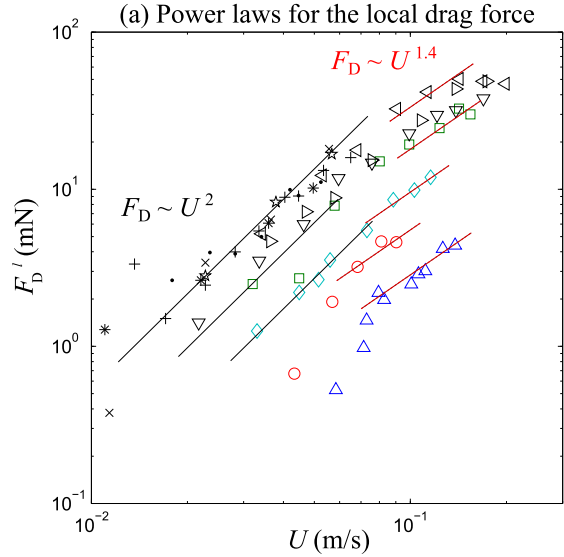

(b) Local and global measurements

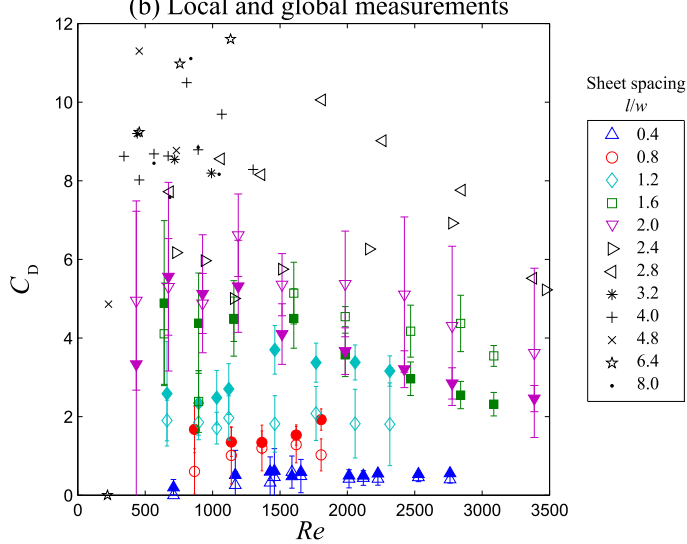

FIG. 4. (a) Log-log representation of the same data as Figure 3(b). The black continuous line shows the trend $F_{\mathrm{D}} \propto \bar{U}^{2}$ for small $\bar{U}$ and the red dashed line $F_{\mathrm{D}} \propto \bar{U}^{2+\mathcal{V}}$ for large $\bar{U}$. (b) Non-dimensional comparison with the global profile drag measured from the pressure loss across the channel (closed symbols). Open symbols represent the local data from individual sheet deflection measurements. 
Since the canopy spacing is uniform and it occupies the entire channel length, it is appropriate to consider that the canopy drag force is uniformly distributed over its length. Thus, for equilibrium,

$$
F_{\mathrm{D}}^{\text {canopy }}=N F_{\mathrm{D}}^{g}=\rho g L \sin \beta A_{\mathrm{c}},
$$

where $F_{\mathrm{D}}^{g}$ is referred to as the global drag force experienced by individual sheets in the canopy and $N=L / \ell$ is the number of sheets in the canopy, which is about 250 for the densest canopy. It is pointed out here that this is a straightforward drag measurement as long as the canopy is sufficiently dense. However, when the sheets are sparsely distributed, the measurement is less accurate as the corresponding channel pressure drop is negligibly small.

This independent drag measurement is also studied for various flow velocities $\bar{U}$ and canopy densities $\ell$. The results are presented in Figure 4(b) which compares the drag force deduced by both methods (local, open symbols; global, closed symbols). Both measurements are of the same order of magnitude considering the measurement precision (error bars are shown for both series on the figure).

In the following, index " $g$ " and " $l$ " are used to distinguish the values obtained from the global and local measurements, respectively. Depending on the two techniques, one can compute $C_{D}^{l}$ the local drag coefficient based on the drag force measured via the sheet deflection and $C_{D}^{g}$ is the global drag coefficient. If the drag coefficient is to be independent of the water velocity then $h_{\mathrm{s}}$, the height of the reconfigured sheet subjected to a water flow (see right inset Fig. 1), should be a function only of $\bar{U}$ and the sheet spacing $\ell$. Therefore, in the following, the effect of canopy density on the sheet reconfiguration is studied to determine the relation between the Vogel exponent $\mathcal{V}$ and the canopy properties.

\section{CANOPY RECONFIGURATION}

To study the reconfiguration of the canopy, it is convenient to use the non-dimensional Cauchy number $C_{Y}$ which measures the sheet deformation as a response for a given incident stress (see Eq. (4)) and the reconfiguration number $\mathcal{R}$ which measures the drag-reduction ratio, as given by the ratio of the drag on a flexible object to the drag on the same object if it were rigid. As the drag force is proportional to the frontal area of the sheet that is facing the flow, the reconfiguration number is then

$$
\mathcal{R} \equiv \frac{h_{\mathrm{s}}}{h}=\int_{0}^{1} \sin \varphi(\tilde{s}) \mathrm{d} \tilde{s} .
$$

Using simultaneous global drag measurements and local deflection measurements as in Fig. 2, the global Cauchy number $C_{\mathrm{Y}}^{g}=C_{\mathrm{D}}^{g} \rho w h^{3} \bar{U}^{2} / 2 E I$ and the sheet reconfiguration number can be calculated independently. They are displayed in Fig. 5(a) for a range of Reynolds numbers $R e_{\mathrm{P}}=$ $400-2200\left(\operatorname{Re}_{\mathrm{P}}=w \bar{U} / v\right.$, where $v$ is the water kinematic viscosity $)$ and canopy configurations corresponding to three different sheet spacings and two sheet widths.

When the Cauchy number is small $\left(C_{\mathrm{Y}}^{g} \ll 1\right)$, there is very little sheet reconfiguration. However, when the global Cauchy number is about $O(1)$, there is a steep decrease in reconfiguration number $\mathcal{R}$. For given elastic properties, the Cauchy number is simply proportional to the drag force. Thus, at small $C_{\mathrm{Y}}^{g}$, the drag force is not sufficiently large to produce any remarkable bending of the PVC sheets in a canopy. In this case, it can be easily shown from the bending beam model (Eq. (4)) that $(1-\mathcal{R}) \propto\left(C_{\mathrm{Y}}^{g}\right)^{2}$ as depicted in Fig. 5(b) where all data collapse on a power-law for $C_{\mathrm{Y}} \ll 1$ (even for $C_{\mathrm{Y}}^{g} \sim O(1)$ ). On the other hand, at large $C_{\mathrm{Y}}^{g}$, the drag force overtakes the elastic rigidity of the PVC sheets and the reconfiguration number $\mathcal{R}$ decreases rapidly.

In Figure 5(a), the experimental data based on the global Cauchy number and the simultaneous deflection measurements are compared to the non-linear flexible model (Eq. (4)) and to the rigid model proposed by de Langre, ${ }^{4}$ where sheets are modelled by rigid cylinders mounted on torsional springs. Both models provide good predictions for the reconfiguration of a sheet. The rigid model ${ }^{4}$ fits the data for small deflections but it over-predicts sheet reconfiguration at large Cauchy numbers, i.e., when the canopy bends sharply. The flexible model which accounts for local sheet bending matches well with data when $C_{\mathrm{Y}}^{g} \sim O(1)$ or greater. 

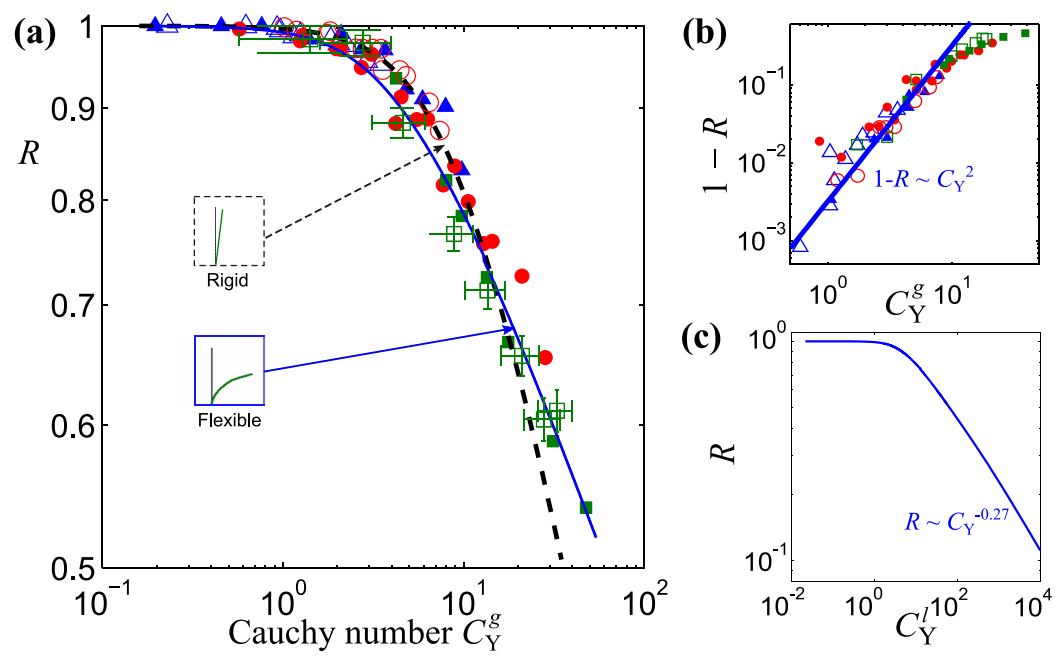

FIG. 5. (a) Reconfiguration curve depicting the reconfiguration number, $\mathcal{R}$, as a function only of the global Cauchy number, $C_{\mathrm{Y}}^{g}=C_{\mathrm{D}}^{g} \rho w h^{3} \bar{U}^{2} / 2 E I$, as computed from $F_{D}^{g}$ via the inclinometer (see Section II C). The closed and open symbols represent two sheet widths ( $w=10$ and $20 \mathrm{~mm}$, respectively) in canopies with various sheet spacings $\ell=32 \mathrm{~mm}$ (olive green square), $16 \mathrm{~mm}$ (red circle), and $8 \mathrm{~mm}$ (blue triangle). For the sake of simplicity, the error bars are shown for only one data series. The dashed and continuous lines denote rigid and flexible bending models, respectively. (b) Evolution of $(1-\mathcal{R})$ against $C_{\mathrm{Y}}$ wherein the solid line is the trend for small Cauchy numbers, $(1-\mathcal{R}) \propto C_{\mathrm{Y}}^{2}$. (c) Deflected beam model (Eq. (4)) showing the trend $\mathcal{R} \propto C_{\mathrm{Y}}^{\alpha}$ at $C_{\mathrm{Y}} \gg 1$.

The reconfiguration curve Fig. 5(c) indicates a power law $\mathcal{R} \propto\left(C_{\mathrm{Y}}^{g}\right)^{\alpha}$ at $C_{\mathrm{Y}} \gg 1$. As suggested by Gosselin et al. ${ }^{40}$ it is, thus, possible to compute the Vogel exponent $\mathcal{V}=2 \alpha$. For all canopy configurations considered here, $\mathcal{V} \simeq-0.6$, which is very close to that for a single fibre under large deformation $(\mathcal{V}=-2 / 3) .{ }^{38,39}$ However, it is larger than that of an artificial leaf folding up in an air flow $(\mathcal{V}=-4 / 3){ }^{43}$ This is simply due to the fact that individual sheets in our canopy configuration are similar to a flexible body that deform predominantly by bending in the flow direction without folding about their edges. In addition, data corresponding to various canopy density fall on the same reconfiguration curve in Figure 5(a). Thus, our study strongly suggests that the canopy density does not influence the Vogel exponent $\mathcal{V}$. Note that this result for a $2 \mathrm{D}$ laterally confined canopy is fundamentally different from that obtained for poro-elastic models of coniferous trees. ${ }^{52}$ In the latter case, the filaments upstream are strongly deformed so that they are almost perpendicular to the incoming flow while the filaments downstream align themselves with the downstream flow. Whereas in the present case, the canopy is distributed evenly throughout the channel's length and so, no particular sheet is exposed differently from the others once a uniform (steady and fully developed) flow is established after the first few sheets. Thus, the porous media seen by a fluid flow over a ball covered-up by flexible filaments are not similar to that of a series of flexible flat plates perpendicular to the flow.

As inferred in Section II, for a given sheet (or a canopy) subjected to a water velocity $\bar{U}$, the sheet drag force is smaller for a denser canopy configuration. It is not straightforward to deduce the effect of sheet spacing on reconfiguration number in Figure 5. Thus, at a given velocity, the canopy density plays an indirect role of reducing sheet reconfiguration via a reduced drag force.

\section{CANOPY DENSITY AND CONFINEMENT}

The drag force on a sheet can be rewritten as $F_{\mathrm{D}}=C_{\mathrm{D}} \frac{1}{2} \rho \bar{U}^{2} w h_{\mathrm{s}}$, where $h_{\mathrm{s}}=\mathcal{R} h \propto \bar{U}^{\mathcal{V}}$ is independent of the canopy spacing $\ell$ (see Section III on reconfiguration). It is therefore expected that the form drag coefficient $C_{\mathrm{D}}$ should be a function of the canopy density. Hence, to further study the effect of canopy density on the drag force and canopy reconfiguration, the effect of the former on the drag coefficient should be explored. Even though the drag force measured via sheet deflection 

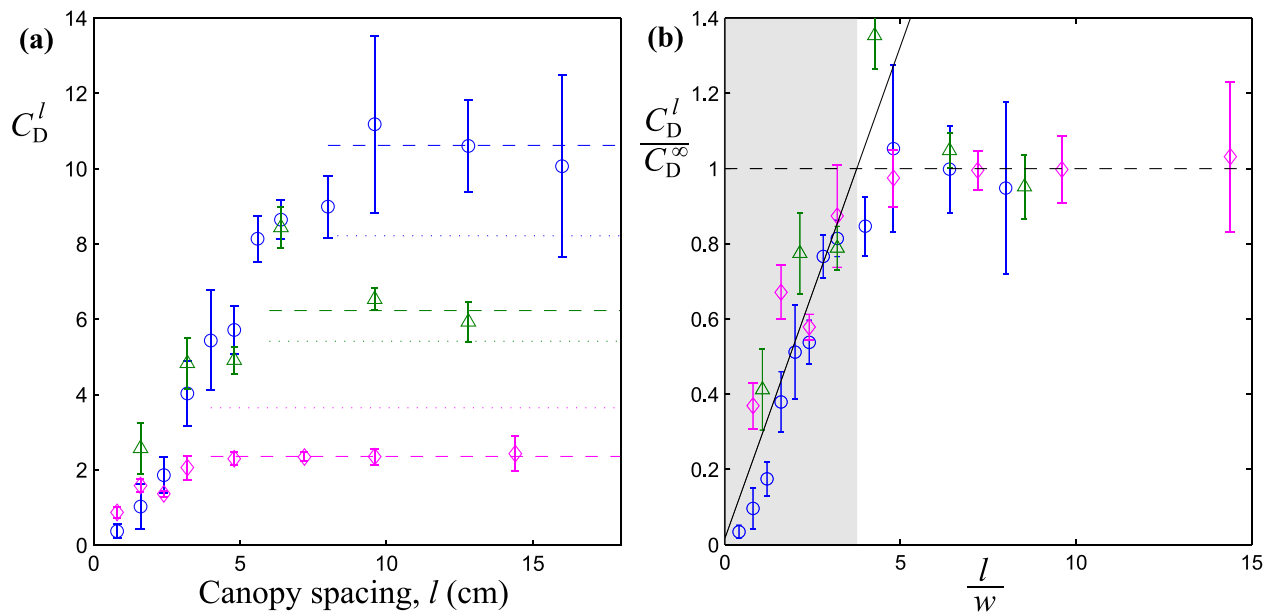

FIG. 6. (a) Profile drag coefficients $C_{\mathrm{D}}^{l}$ of a PVC sheet in a canopy are plotted against the canopy spacing $\ell$ for different sheet widths $w=10 \mathrm{~mm}$ (magenta lozenge), $15 \mathrm{~mm}$ (olive green triangle), and $20 \mathrm{~mm}$ (blue circle). The dashed line is the measured asymptotic value for large spacings, whereas the point line is the same value estimated with Glauert's formula (Eq. (7)). (b) The data are normalized with the asymptotic value for large spacings $C_{\mathrm{D}}^{l, \infty}$ with respect to the non-dimensional canopy spacing $\ell / w$. They show two distinct regimes, namely, a dense regime where the drag coefficient first increases linearly as $\ell / w$ (shaded zone) and an isolated sheet regime wherein it becomes approximatively a constant maximum corresponding to that of an isolated sheet when $\ell>4 w$.

$F_{D}^{l}$ and via an inclinometer $F_{\mathrm{D}}^{g}$ is of the same order of magnitude, only the "local" drag coefficient $C_{\mathrm{D}}^{l}=F_{\mathrm{D}}^{l} / \frac{1}{2} \rho \bar{U}^{2} w h_{s}$ are used in the following for the sake of simplicity. When data corresponding to $C_{D}^{g}$ is used along with $C_{D}^{l}$, there is some quantitative dispersion; nevertheless the conclusions of this section remain the same for both measurements. Figure 6(a) displays $C_{\mathrm{D}}^{l}$ in different canopy configurations. Here, for each configuration, the length of the canopy $L \simeq 2 \mathrm{~m}$ is fixed and only the spacing between each PVC sheet $\ell$ is modified over a range of about $8-160 \mathrm{~mm}$. The error bars for the local drag coefficient come from standard error between $C_{\mathrm{D}}^{l}$ of the ten measured sheet in the canopy. Note that the drag coefficients are much larger than the commonly known values (about 2 , see Vogel ${ }^{32}$ ) at these Reynolds numbers. This is simply due to large confinement effects. ${ }^{38}$

Consider the case when the sheet width is $w=20 \mathrm{~mm}$. $C_{\mathrm{D}}^{l}$ vary first linearly with respect to the canopy spacing $\ell$ before it reaches approximately a constant maximum when $\ell \geqslant 10 \mathrm{~cm}$. The same trend is observed for the other two sheet widths displayed in Figure 6(a) while the critical spacing $\ell_{c}$ at which $C_{\mathrm{D}}^{l}$ saturates is different for each sheet width $w: \ell_{c} \approx 6 \mathrm{~cm}$ for $w=15 \mathrm{~mm}$ and $\ell_{c} \approx 4 \mathrm{~cm}$ for $w=10 \mathrm{~mm}$. An estimation for the drag coefficient $C_{D}^{l, \infty}$ of a laterally confined isolated sheet can be obtained by using the classical Glauert's formula, ${ }^{60}$

$$
C_{D}^{l, \infty} \approx \bar{C}_{D}^{l, \infty}\left[\frac{1+\tau \lambda\left(\frac{w}{b}\right)^{2}}{\left(1-\eta \frac{w}{b}\right)^{2}}\right],
$$

which relates the measured drag coefficient $C_{D}^{l, \infty}$ of a bluff body subjected to lateral confinement with that in an infinite medium $\bar{C}_{D}^{l, \infty}$ via the ratio between the sheet width $w$ and the channel width $b$. Here, $\tau=0.822, \lambda=0.5$, and $\eta=1$ are empirical constants for the case of a flat plate normal to the flow. If we take $\bar{C}_{D}^{l, \infty}=2$ corresponding to a thin plate in an infinite inviscid medium, ${ }^{61}$ Glauert's formula (Eq. (7)) provides a decent prediction for the experimental values shown in Figure 6(a).

These data are now plotted (see Figure 6(b)) in terms of the normalized drag coefficient $C_{\mathrm{D}}^{l} / C_{\mathrm{D}}{ }^{l, \infty}$ against the non-dimensional canopy spacing $\varepsilon=\ell / w$. Note that this single sheet drag coefficient depends on the lateral confinement and then on the sheet width $w$. It is striking that all data fall almost along a unique curve. Two dominant features are observed: (1) $C_{\mathrm{D}}^{l} \propto(\ell / w) C_{\mathrm{D}}^{l, \infty}$ as long as the spacing between sheets is smaller than the critical spacing $\ell_{c}$ (dense regime) and (2) $C_{\mathrm{D}}^{l}$ is independent of the canopy spacing $\ell$ and is equal to that of an isolated sheet (isolated sheet regime). Therefore, a sheet inside a canopy should experience a smaller drag force $F_{\mathrm{D}}$ than its 
rigid counterpart via two drag reduction techniques: on one hand, the elastic reconfiguration which results in a sub-linear speed-drag dependence $\left(F_{\mathrm{D}} \propto \bar{U}^{2+\mathcal{V}}\right)$ and on the other hand, the sheltering effect due to its neighbours wherein the drag force $F_{\mathrm{D}}$ decreases linearly with the spacing between sheets $\ell$, as long as $\ell \leqslant \ell_{c}$.

Since the length of the recirculation zone behind a flat plate is about 4-5 times its width, it is expected that the presence of a sheet influences its neighbours as long as $\ell \leqslant 4 w$. In fact, Figure 6 indicate that the critical canopy spacing is indeed $\ell_{c} \approx 4 w$. When $\ell<\ell_{c}$ individual sheets are, thus, expected to be sheltered so effectively by their neighbours' recirculation zone that they are not exposed to the incoming water velocity $\bar{U}$ but to a very negligible water flow perpendicular to them. This implies that in the dense regime, due to the low momentum wake between the sheets, the canopy behaves like single continuous flexible media. The force acting on such a canopy should then be equal to the product of the drop in pressure across the channel $\Delta P$ and the reconfigured frontal area of the canopy $A_{\mathrm{s}}$. For an inviscid flow, the former is proportional to the dynamic pressure $\frac{1}{2} \rho \bar{U}^{2}$ whereas the latter is dependent on water velocity via the Vogel exponent as $\bar{U}^{\mathcal{V}}$. Therefore, the total drag $F^{c a n}$ on the whole canopy must be $F^{c a n} \propto \bar{U}^{2+\mathcal{V}}$. But the net force $F^{c a n}$ on the canopy is equal to the sum of all the forces acting on each sheet in it. Given that the sheets occupy the entire length of the channel and, also, the canopy is quasi-two dimensional, the force per unit length should be homogeneous across the canopy when the flow rate is constant across it. So, $F^{c a n}=N F_{\mathrm{D}}$ where $F_{\mathrm{D}}$ is the sheet and $N=(L / \ell)$ is the number of sheets in the canopy configuration. Therefore, the drag force $F_{\mathrm{D}}$ should be linearly proportional to the spacing, $\ell$. On the other hand, if the sheets are not exposed to their neighbours' recirculation zone $\ell>\ell_{c}$, each one of them is left alone to face the incoming water flow at about $\bar{U}$. In this case, only drag reduction via reconfiguration occurs and the drag force is simply independent of the sheet spacing. These results are expected to be valid for a non-confined array of sheets, insofar as the dependence in channel width is included in the modified value of the plateau drag coefficient $C_{\mathrm{D}}^{\infty}$.

Experimental results from the reconfiguration study of simple quasi- $2 D$ confined canopies suggest that $\mathcal{V}$ is independent of the canopy density. Since the Vogel exponent $\mathcal{V}$ should depend on the incident velocity profile ${ }^{57}$ this should arise from the fact that the velocity profile in the wake of a vertical plate is self-similar with respect to the stream-wise direction. ${ }^{61}$ However, in real-life situations, dense vegetation is often irregular wherein the size of individual plants/trees inside a canopy and also the spacing between them should differ considerably. Nonetheless, the Vogel exponent $\mathcal{V}$ is expected to be approximatively -0.6 as in our case because the wake velocity profile should not show any qualitative difference except maybe when the flow is turbulent. In fact, it is known that $\mathcal{V}$ varies only a little about -0.6 for most of the field observations. ${ }^{19,57}$

\section{CONCLUSION}

A $2 \mathrm{~m}$ long canopy of PVC sheets subjected to a laterally confined open channel flow is studied, in order to determine the effect of sheet density on the drag reduction mechanisms. The canopy is observed to behave as a continuous flexible object where all the sheets show approximatively the same deflection when a uniform water flow is maintained. Two independent methods were performed to make sheet deflection and drag measurements on various canopy configurations: (1) individual sheet deflection and the respective "local" sheet drag force $F_{\mathrm{D}}^{l}$; and (2) a global drag force $F_{\mathrm{D}}^{g}$ on a sheet in a canopy is computed via the global force balance on an inclinable water channel. It is observed that $F_{\mathrm{D}}^{l}$ and $F_{\mathrm{D}}^{g}$ are about the same order of magnitude over various Reynolds numbers and canopy densities. Then, by comparing the global Cauchy number $\left(C_{\mathrm{Y}}^{g}=C_{\mathrm{D}}^{g} \rho w h^{3} \bar{U}^{2} / 2 E I\right)$ based on the measured $F_{\mathrm{D}}^{g}$ and the local deflection measurements, the dimensionless reconfiguration number representing the canopy deflection is shown to be a function only of the Cauchy number, independent of the spacing between the sheets. It is, therefore, concluded that each sheet in a canopy behaves like a single flexible object provided that an appropriate drag coefficient is defined, which takes into account the canopy density; the Vogel exponent $\mathcal{V}$ is independent of the canopy density.

For a given sheet width $w$, if the sheet spacing $\ell \leq 4 w$, the drag coefficient $C_{\mathrm{D}}^{l}$ of an individual sheet decreases when the canopy becomes denser. Measurements indicate that $C_{\mathrm{D}}^{l}$ varies linearly 
with the spacing between the sheet $\ell$ so that $C_{\mathrm{D}}^{l} \propto(\ell / w) C_{\mathrm{D}}^{l, \infty}$ where $C_{\mathrm{D}}^{l, \infty}$ is the drag coefficient of an isolated sheet (measured from its deflection). Within this limit, the drag force $F_{\mathrm{D}}$ is simply proportional to $\ell$. Beyond this limit $(\ell \geq 4 w)$, the drag force does not depend on the canopy density and is equal to that of an isolated flexible sheet. This observation is substantially different from common assumptions in theoretical models ${ }^{21,23,48}$ that try to describe coherent structures arising from fluid-structure interactions in submerged vegetation canopies. Most canopies are not as simple as the ones considered here. Therefore, it is hoped that these new results for an array of flexible plates perpendicular to the flow would encourage further investigations on the role of canopy density in more complex flexible, porous media analogous to aquatic vegetation.

\section{ACKNOWLEDGMENTS}

The authors are grateful to Olivier Gain and Laurent David from IMP laboratory (Polytech'Lyon) for the sheets rigidity measurements. The authors also acknowledge Amandine Pâquet and Stéphane Martinez for their technical support.

${ }^{1}$ W. O. Ree and V. J. Palmer, Flow of Water in Channels Protected by Vegetative Linings (US Department of Agriculture, Washington, D.C., 1949).

${ }^{2}$ H. M. Nepf, "Flow and transport in regions with aquatic vegetation," Annu. Rev. Fluid Mech. 44, 123-142 (2012).

${ }^{3}$ S. M. Haslam, River Plants: The Macrophytic Vegetation of Watercourses (CUP Archive, 1978).

${ }^{4}$ E. de Langre, "Effects of wind on plants," Annu. Rev. Fluid Mech. 40(1), 141-168 (2008).

${ }^{5}$ G. Bornette and S. Puijalon, "Response of aquatic plants to abiotic factors: A review," Aquat. Sci. 73(1), 1-14 (2011).

${ }^{6}$ C. Sanchez, H. Arribart, and M. M. Giraud Guille, "Biomimetism and bioinspiration as tools for the design of innovative materials and systems," Nat. Mater. 4(4), 277-288 (2005).

${ }^{7}$ B. Bhushan, "Biomimetics: Lessons from nature-an overview," Philos. Trans. R. Soc., A 367(1893), 1445-1486 (2009).

${ }^{8}$ J. Finnigan, "Turbulence in plant canopies," Annu. Rev. Fluid Mech. 32, 519-571 (2000).

${ }^{9}$ P. Naden, P. Rameshwaran, O. Mountford, and C. Robertson, "The influence of macrophyte growth, typical of eutrophic conditions, on river flow velocities and turbulence production," Hydrol. Processes 20(18), 3915-3938 (2006).

${ }^{10}$ H. M. Nepf, "Hydrodynamics of vegetated channels," J. Hydraul. Res. 50(3), 262-279 (2012).

${ }^{11}$ R.-M. Li and H. W. Shen, "Effect of tall vegetations on flow and sediment," J. Hydraul. Div. 99(5), 793-814 (1973).

${ }^{12}$ F. Lopez and M. Garcia, "Open-channel flow through simulated vegetation: Suspended sediment transport modeling," Water Resour. Res. 34(9), 2341-2352, doi:10.1029/98WR01922 (1998).

13 J. Järvelä, J. Aberle, A. Dittrich, H. P. Rauch, and I. Schnauder, "Flow-vegetation-sediment interaction: Research challenges," in River Flow 2006, edited by R. M. L. Ferreira, E. C. T. L. Alves, J. G. A. B. Leal, and A. H. Cardoso (Taylor \& Francis, 2006), Vol. 2, pp. 2017-2026.

${ }^{14}$ N. Kouwen and M. Fathi-Moghadam, "Friction factors for coniferous trees along rivers," J. Hydraul. Eng. 126(10), 732-740 (2000).

${ }^{15}$ H. M. Nepf, "Drag, turbulence, and diffusion in flow through emergent vegetation," Water Resour. Res. 35(2), 479-489, doi:10.1029/1998WR900069 (1999).

${ }^{16}$ F. C. Wu, H. W. Shen, and Y. J. Chou, "Variation of roughness coefficients for unsubmerged and submerged vegetation," J. Hydraul. Eng. 125(9), 934-942 (1999).

${ }^{17}$ N. Kouwen and T. E. Unny, "Flexible roughness in open channels," J. Hydraul. Div. 99, 713-728 (1973).

${ }^{18}$ M. Righetti and A. Armanini, "Flow resistance in open channel flows with sparsely distributed bushes," J. Hydrol. 269(1-2), 55-64 (2002).

${ }^{19}$ E. de Langre, "Methodological advances in predicting flow-induced dynamics of plants using mechanical-engineering theory," J. Exp. Biol. 215, 914-921 (2013).

${ }^{20}$ M. Ghisalberti and H. M. Nepf, "Mixing layers and coherent structures in vegetated aquatic flows," J. Geophys. Res. 107, 3-1-3-11, doi:10.1029/2001JC000871 (2002).

${ }^{21}$ C. Py, E. de Langre, and B. Moulia, "A frequency lock-in mechanism in the interaction between wind and crop canopies," J. Fluid Mech. 568, 425-449 (2006).

${ }^{22}$ S. M. Cameron, V. I. Nikora, I. Albayrak, O. Miler, M. Stewart, and F. Siniscalchi, "Interactions between aquatic plants and turbulent flow: A field study using stereoscopic PIV," J. Fluid Mech. 732, 345-372 (2013).

${ }^{23}$ R. Singh, M. M. Bandi, A. Mahadevan, and S. Mandre, "Linear stability analysis for monami in a submerged seagrass bed," J. Fluid Mech. 786, R1 (2016).

${ }^{24}$ S. G. Monismith, "Hydrodynamics of coral reefs," Annu. Rev. Fluid Mech. 39, 37-55 (2007).

${ }^{25}$ M. Luhar and H. M. Nepf, "Wave-induced dynamics of flexible blades," J. Fluids Struct. 61, 20-41 (2016)

${ }^{26}$ J. S. Madin and S. R. Connolly, "Ecological consequences of major hydrodynamic disturbances on coral reefs," Nature 444, 477-480 (2006).

${ }^{27}$ D. Lopez, S. Michelin, and E. de Langre, "Flow-induced pruning of branched systems and brittle reconfiguration,” J. Theor. Biol. 284, 117-124 (2011).

${ }^{28}$ R. Nathan, G. Katul, H. Horn, S. Thomas, and R. Oren, "Mechanisms of long-distance dispersal of seeds by wind," Nature 418, 409-413 (2002).

${ }^{29}$ R. Nathan and G. Katul, "Foliage shedding in deciduous forests lifts up long-distance seed dispersal by wind," Proc. Natl. Acad. Sci. 102, 8251-8256 (2005). 
${ }^{30}$ S. Vogel, "Drag and flexibility in sessile organisms," Am. Zool. 24(1), 37-44 (1984).

${ }^{31}$ S. Vogel, "Drag and reconfiguration of broad leaves in high winds," J. Exp. Bot. 40(217), 941-948 (1989).

${ }^{32}$ S. Vogel, Life in Moving Fluids: The Physical Biology of Flow (Princeton University Press, 1994).

${ }^{33}$ T. Barois and E. de Langre, "Flexible body with drag independent of the flow velocity," J. Fluid Mech. 735, R2 (2013).

${ }^{34}$ K. Sand-Jensen, "Drag and reconfiguration of freshwater macrophytes," Freshwater Biol. 48(2), 271-283 (2003).

${ }^{35}$ O. Speck and H.-C. Spatz, "Damped oscillations of the giant reed Arundo donax (Poaceae)," Am. J. Bot. 91(6), 789-796 (2004).

${ }^{36}$ M. Rudnicki, S. J. Mitchell, and M. D. Novak, "Wind tunnel measurements of crown streamlining and drag relationships for three conifer species," Can. J. For. Res. 34(3), 666-676 (2004).

${ }^{37}$ S. Vollsinger, S. J. Mitchell, K. E. Byrne, M. D. Novak, and M. Rudnicki, "Wind tunnel measurements of crown streamlining and drag relationships for several hardwood species," Can. J. For. Res. 35(5), 1238-1249 (2005).

${ }^{38}$ S. Alben, M. J. Shelley, and J. Zhang, "Drag reduction through self-similar bending of a flexible body," Nature 420(6915), 479-481 (2002).

${ }^{39}$ S. Alben, M. J. Shelley, and J. Zhang, "How flexibility induces streamlining in a two-dimensional flow," Phys. Fluids 16(5), 1694 (2004).

${ }^{40}$ F. P. Gosselin, E. de Langre, and B. A. Machado-Almeida, "Drag reduction of flexible plates by reconfiguration,” J. Fluid Mech. 650, 319-341 (2010).

${ }^{41}$ J. T. Dijkstra and R. E. Uittenbogaard, "Modeling the interaction between flow and highly flexible aquatic vegetation," Water Resour. Res. 46(12), W12547, doi:10.1029/2010WR009246 (2010).

${ }^{42}$ M. Luhar and H. M. Nepf, "Flow-induced reconfiguration of buoyant and flexible aquatic vegetation," Limnol. Oceanogr. 56(6), 2003-2017 (2011).

${ }^{43}$ L. Schouveiler and A. Boudaoud, "The rolling up of sheets in a steady flow," J. Fluid Mech. 563, 71-80 (2006).

${ }^{44}$ I. Albayrak, V. Nikora, O. Miler, and M. O. Hare, "Flow-plant interactions at a leaf scale: Effects of leaf shape, serration, roughness and flexural rigidity," Aquat. Sci. 74(2), 267-286 (2012).

${ }^{45}$ R. D. Blevins, Flow-Induced Vibration (Van Nostrand Reinhold Co., New York, 1977).

${ }^{46}$ A. S. Thom, "Momentum absorption by vegetation,” Q. J. R. Meteorol. Soc. 97, 414-428 (1971).

${ }^{47}$ H. M. Nepf and E. R. Vivoni, "Flow structure in depth-limited, vegetated flow," J. Geophys. Res. 105(C12), 28547-28557, doi:10.1029/2000JC900145 (2000).

${ }^{48}$ F. P. Gosselin and E. de Langre, "Destabilising effects of plant flexibility in air and aquatic vegetation canopy flows," Eur J. Mech., B: Fluids 28, 271-282 (2009).

${ }^{49}$ M. R. Hoffmann, "Application of a simple space-time averaged porous media model to flow in densely vegetated channels," J. Porous Media 7(3), 183 (2004).

${ }^{50}$ O. Doaré, B. Moulia, and E. de Langre, "Effect of plant interaction on wind-induced crop motion,” J. Biomech. Eng. 126(2), 146-151 (2004).

${ }^{51}$ J. Favier, A. Dauptain, D. Basso, and A. Bottaro, "Passive separation control using a self-adaptive hairy coating," J. Fluid Mech. 627, 451-483 (2009).

${ }^{52}$ F. P. Gosselin and E. de Langre, "Drag reduction by reconfiguration of a poroelastic system," J. Fluids Struct. 27(7), 1111-1123 (2011).

${ }^{53}$ Y. Brunet, J. J. Finnigan, and M. R. Raupach, "A wind tunnel study of air flow in waving wheat: Single-point velocity statistics," Boundary-Layer Meteorol. 70, 95-132 (1994).

${ }^{54}$ M. D. Novak, J. S. Warland, A. L. Orchansky, R. Ketler, and S. Green, "Wind tunnel and field measurements of turbulent flow in forests. Part I: Uniformly thinned stands,” Boundary-Layer Meteorol. 95, 457-495 (2000).

${ }^{55}$ C. H. Peterson, R. A. Luettich, F. Micheli, and G. A. Skilleter, "Attenuation of water flow inside seagrass canopies of differing structure," Mar. Ecol.: Prog. Ser. 268, 81-92 (2004).

${ }^{56}$ D. Poggi, A. Porporato, L. Ridolfi, J. D. Albertson, and G. G. Katul, "The effect of vegetation density on canopy sub-layer turbulence," Boundary-Layer Meteorol. 111, 565-587 (2004).

${ }^{57}$ T. Leclercq and E. de Langre, "Drag reduction by elastic reconfiguration of non-uniform beams in non-uniform flows," J Fluids Struct. 60, 114-129 (2016).

${ }^{58}$ L. Chevalier, Mécanique des Systèmes et des Milieux Déformables (Ellipses Edition, 1994).

${ }^{59}$ D. M. Temple, K. M. Robinson, R. M. Ahring, and A. G. Davis, Stability Design of Grasslined Open Channels Vol. 667 (US Department of Agriculture, Agricultural Research Service, 1987).

${ }^{60}$ H. Glauert, "Wind tunnel interference on wings, bodies and airscrews," Technical Report 1566, DTIC Document, 1933.

${ }^{61}$ G. K. Batchelor, An Introduction to Fluid Dynamics (Cambridge University Press, 2000). 